\title{
Anabases
}

ANABASES Traditions et réceptions de l'Antiquité

$11 \mid 2010$

Varia

\section{Dominique LENFANT (éd.), Athénée et les fragments} d'historiens

Sylvie Rougier-Blanc

\section{OpenEdition}

Journals

Édition électronique

URL : http://journals.openedition.org/anabases/905

DOI : 10.4000/anabases.905

ISSN : 2256-9421

\section{Éditeur}

E.R.A.S.M.E.

\section{Édition imprimée}

Date de publication : 1 mars 2010

Pagination : 267-269

ISSN : 1774-4296

\section{Référence électronique}

Sylvie Rougier-Blanc, "Dominique lenfant (éd.), Athénée et les fragments d'historiens », Anabases [En ligne], 11 | 2010, mis en ligne le 01 septembre 2011, consulté le 22 septembre 2020. URL : http:// journals.openedition.org/anabases/905; DOI : https://doi.org/10.4000/anabases.905

Ce document a été généré automatiquement le 22 septembre 2020.

(c) Anabases 


\title{
Dominique LENFANT (éd.), Athénée et les fragments d'historiens
}

\author{
Sylvie Rougier-Blanc
}

\section{RÉFÉRENCE}

Dominique LENFANT (éd.), Athénée et les fragments d'historiens, actes du colloque de Strasbourg (16-18 juin 2005), Paris, De Boccard, 2007, Études d'archéologie et d'histoire ancienne, Université Marc Bloch, $474 \mathrm{p}$.

43 euros / ISBN 978-2-7018-0230-5.

1 Dominique Lenfant publie les actes du colloque de Strasbourg sur les fragments d'historiens chez Athénée de Naucratis organisé en juin 2005. Athénée (fin II ${ }^{\mathrm{e}}$-début III ${ }^{\mathrm{e}}$ s. ap. J.-C.) met en scène des savants (sophistai) qui, au cours d'un banquet, multiplient les allusions sur les manières de table et citent près de 2500 ouvrages, principalement de comiques et d'historiens, pour la plupart aujourd'hui disparus. Le volume propose de riches annexes dont un descriptif de la structure de l'œuvre très utile pour se repérer. Suivent une bibliographie complète et de nombreux indices. Nul doute qu'il s'agisse là d'un excellent outil de travail.

2 Le propos n'est pas nouveau mais l'angle d'approche privilégie la méthode d'Athénée en trois grandes parties : la première consacrée aux historiens conservés, la deuxième aux historiens non conservés. Il s'agit d'élaborer une méthode d'analyse des citations. La troisième partie, «fragments non historiques ", est moins bien rattachée au propos. De plus, la notion de fragment appliquée aux Deipnosophistes est discutable car elle insiste sur la discontinuité : comme le propose Christophe Bréchet (p.321-322, n. 1) « extrait ou citation» serait plus juste et permettrait de placer la question de la réception au second plan de la réflexion. On regrettera aussi que chaque article soit alourdi par une reprise des principes de définition des critères de citations sans toujours tenir compte des acquis des articles précédents : c'est là bien souvent le propre des actes de colloque que de juxtaposer des points de vue qui se recoupent. Une brève 
synthèse des résultats et des acquis méthodologiques en fin de chapitre aurait été bienvenue. Une conclusion générale aurait aussi permis de mieux cerner les avancées du colloque et d'éviter l'impression d'éclatement.

3 Les deux premières contributions insistent sur le projet athénéen: John Wilkins (p. 29-39) montre que si Athénée cite les historiens canoniques, il opte pour l'histoire « sympotique ». Il laisse entendre que les citations d'historiens, extrêmement orientées et filtrées, participent activement à un projet cohérent et unifié. Giuseppe Zecchini dans «Athénée et les historiens: un rapport indirect»(p.19-28) conclut aussi qu'Athénée puise chez les historiens du matériau pour des questions d'histoire de la société plutôt que d'histoire proprement dite (militaire ou politique).

Cette approche est en partie confirmée par les analyses détaillées des chapitres suivants, notamment celles de Dominique Lenfant (p.43-72) et de Christine Maisonneuve (p. 73-106) respectivement sur les citations d'Hérodote et de Xénophon. Elles répertorient les différents types de citations et les comparent aux textes connus par la tradition. Athénée ne saurait suffire à nous donner un aperçu pertinent de leur production historique. Elles insistent sur la réorientation sémantique, voire la distorsion qu'Athénée fait subir au texte cité. On ne peut en effet confondre citation textuelle (fidèle au texte d'origine) et citation littérale (qui reproduit la littéralité exacte) comme le soulignent Antonio L. Chavez Reino et Gabriella Ottone à propos de Théopompe, (p. 165). L'enjeu est de comprendre les modalités du détournement. C'est bien ce que proposent de nombreuses contributions, plus particulièrement les analyses de Pascale Giovannelli-Jouanna (p. 215-237) sur Douris de Samos, de Guido Schepens, (p. 239-261) sur Philarque, et de Dino Ambaglio (p. 109-116) sur Hellanicos. Le Naucratite nous livre des informations capitales sur les historiens perdus, leurs usages à son époque, leurs dates, plus qu'un relevé fiable et un aperçu représentatif de leurs productions. Il nous dévoile aussi ses préférences. Un certain nombre de contributions exploite cet aspect des Deipnosophistes. Par recoupement et avec beaucoup de prudence, Pascal Payen (p. 191-214) replace les "fragments" de Charès de Mytilène dans le contexte des genres de la littérature de banquet (notamment les aitiai). Il fait partie des historiens non rhétoriques des $\mathrm{IV}^{\mathrm{e}}-\mathrm{III}^{\mathrm{e}} \mathrm{s}$. av. J.-C., dont l'œuvre est admirée, connue et respectée. Edmond Lévy (p. 277-289) étudie dans une même perspective Sosibios de Sparte qu'il distingue de ses homonymes, et qu'il définit comme « un antiquaire » de la période romaine ( $\mathrm{e}^{\mathrm{er}}$ s. av. $-\mathrm{e}^{\mathrm{er}}$ s. ap. J.-C.). Giovanni Parmeggani ( $\mathrm{I}$ fragmenti di Eforo nei Deipnosophistai di Ateneo », p. 117-137), en étudiant les citations de l'historien du $\mathrm{IV}^{\mathrm{e}} \mathrm{s}$. av.J.-C., montre que les choix d'Athénée peuvent révéler aussi bien les convictions de l'auteur que les lectures à la mode à la fin du $\mathrm{II}^{\mathrm{e}} \mathrm{s}$. Antonio L. Chavez Reino et Gabriella Ottone (p. 139-174), posent la question des sources d'information et de la méthode de travail d'Athénée à propos de Théopompe. Jan Bollansée (p. 175-189) envisage notamment des compilations des constitutions aristotéliciennes pour expliquer la présence marginale des Politeiai chez Athénée. De même qui dit citation longue ne dit pas nécessairement citation de première main, comme le montre Virgilio Costa (p. 263-275) pour l'historien athénien Philochore (340-262 av. J.-C.). Katherine Clarke (p. 291-302) s'appuie sur la concentration de certaines citations de Posidonios et l'utilisation de sections privilégiées pour envisager un système de fiches de lectures ou d'extraits. Cette hypothèse mériterait d'être approfondie, notamment pour d'autres historiens, tout comme l'idée que l'intérêt d'Athénée pour Posidonios repose plus sur des affinités de méthode que de thématique. 
5 La dernière partie du volume, consacrée aux fragments non historiques, moins bien rattachée à l'ensemble, contribue à éclater le propos. On peut néanmoins retenir avec Caroline Magdeleine («les fragments médicaux chez Athénée », p. 355-379) qu’Athénée fait preuve du même désintérêt pour la discipline médicale que pour la véracité historique, alors qu'Homère et Platon connaissent un traitement particulier. Christophe Bréchet (p. 321-340) s'attache à souligner la « matière homérique » connue des convives et d'Athénée lui-même et le traitement particulier dont elle fait l'objet. L'analyse de David Bouvier (p.305-319) cherche à montrer comment se construit l'Homère dont Athénée a besoin, c'est-à-dire celui qui enseigne les bonnes manières de table. Le travail de réinterprétation et de distorsion de la source mis en œuvre pour les citations d'historiens est aussi visible pour Homère mais à plus grande échelle (le "grand livre »), et par le travail critique de l'exégèse. Platon aussi occupe une place centrale chez Athénée. Luciana Romeri aborde l'ensemble des références pour montrer que le Naucratite utilise le philosophe comme il le fait des historiens (p. 341-354) mais avec une hostilité qui dépasse la simple critique historique.

6 Ce volume offre donc un riche éventail des usages de la citation et des historiens chez Athénée. Cependant pour apprécier le projet d'Athénée dans sa totalité et comprendre sa méthode, il est aussi indispensable d'aborder l'œuvre en continu (si indigeste soitelle) et de quitter la logique des « fragments » pour chercher la logique du discours.

\section{AUTEURS}

\section{SYLVIE ROUGIER-BLANC}

Université de Toulouse (UTM)

srougier-blanc@neuf.fr 